\title{
Context-Matching Method as a Transformation Paradigm Between Position and Location Domains in Location-Based Services
}

\author{
Oliver Jukić $^{1^{*}}$, Nenad Sikirica ${ }^{2}$, Teodor Iliev ${ }^{3}$, and Darko Špoljar ${ }^{2}$ \\ ${ }^{1}$ Virovitica University of Applied Sciences, Virovitica, Croatia, \\ ${ }^{2}$ Krapina University of Applied Sciences, Krapina, Croatia \\ ${ }^{3}$ University of Ruse, Department of Telecommunications, Ruse, Bulgaria \\ *E-mail of corresponding author: oliver.jukic@vuv.hr
}

\begin{abstract}
A two-domain approach involving position and location has been introduced to provide a formal description needed for optimal organization of information processing for utilization in Location-Based Services development and operation. This paper proposes the transformation that connects the two domains (position and location), outlines its formal description, and validates the concept using the Context-Matching method as a paradigm.
\end{abstract}

Received 30 August 2021

Accepted 24 September 2021

Published 22 December 2021

Keywords: location-based service, domain transformation.

\section{Introduction}

Location-Based Services are telecommunication services that assist the natural human inclination towards orientation and navigation. In order to provide a formal model of the LBS development and therefore facilitate the LBS development and operation [1]. The authors in [2] proposed the two-domain approach, that distinguishes various phases of the LBS development in position and location domains, respectively.

Here we advance the approach with a proposal for a formal description of the transformation between the two domains using spatial statistics [3] and statistical learning [4], and spatial information fusion [5], and demonstrate its validity with the Context- (Map-) Matching method as a paradigm. A practical realisation is conducted in the open-source R environment for statistical computing.

\section{Problem description and research background}

Navigation, orientation, and individual and group mobility have been proven to be one of the pillars of human civilization and survival. The mobility assistive services of modern Information and Communication Technology (ICT) systems gain constant attraction from both technology and business perspectives. The family of mobility assistive services based on public mobile networks and their services (including the mobile internet, Intelligent Transport Systems, and Internet of Things) is known as Location-Based Services (LBS), due to relation to both position in space and relations with surrounding objects.

In [2] was proposed a new LBS model, related to the architecture of public mobile networks, that allows for establishment of generic LBS Quality of Service (QoS)

(C) The Author(s) 2021. Published by CIEES Association Press. This work is licensed under a Creative Commons Attribution-NonCommercialNoDerivs 4.0 Generic License. description with the QoS guarantees to the LBS users defined in measurable manner. The model has elevated Location-Based Services acceptance and proliferation in the Information and Communication Technologies (ICT) market, becoming a widely utilised class of services.

The two-domains LBS information model distinguishing between the two essential realisations of the place of existence description: position and location, and the means for their determination. In the two-domain LBS information model, position and location, as well as positioning and localisation as the means for their respective determination, are defined as follows [2].

Position is a description of a place where a mobile object resides in material world (physical landscape), providing it is given in an unambiguous manner using the common reference frame $[6,7]$. A position is to be estimated by measurements in material world, using methods based on observation of values of dedicated parameters of physical processes, such as: satellite navigation (GPS, Glonass, GNSS etc.), network-based positioning (Cell-ID, E-TOA, RSS etc.) or the others [7, $8,9]$. Method of that kind is proposed to be referred to as a positioning method. The processes of position-related information processing and management are considered to be conducted in position domain $[2,10]$.

Location is defined as a description of a place where a mobile object resides in contextual world (information/location landscape), providing it is given in an unambiguous fashion using the common location (information) landscape [2]. In analogy with the definition of position, location should be defined in relation to a certain common frame, here firmly related to context [2].

Every method or procedure aiming to place an object in contextual world referring to contextual relations with neighbouring objects in information/location landscape, will be referred to as a localisation method [2]. The processes of location-related information processing and management are considered to be conducted in location domain [2]. 
The information processing for LBS appears to be more feasible and efficient to perform in location domain [3]. The information analysis and modelling in location domain enables: (i) position estimation improvement, (ii) detection and identification of contextual relations, (iii) generalised spatial analysis and predictive modelling, among the other benefits [3]. Earlier developments have established the LBS framework. Here we present the formalised view on the role and the features of the transformation connecting the two domains using spatial statistics [3] and statistical learning [4], and spatial information fusion [5].

\section{Transformation between position and location domains}

The transition between the two domains is to be performed using the appropriate transformation. The transformation applies to a descriptive set of attributes in one domain and transforms them into the equivalent set of attributes in the other one (Figure 1), allowing for an efficient and feasible information processing as a foundation for LBS delivery.
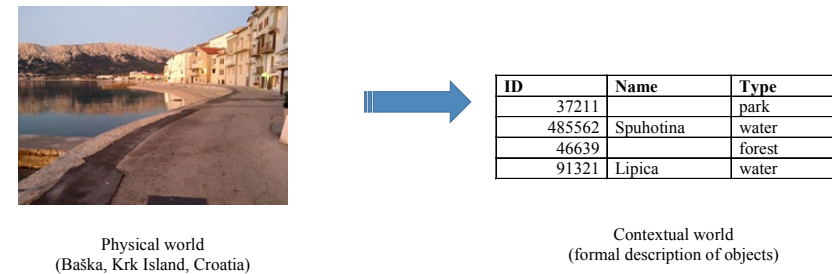

Figure 1. Physical world vs Contextual world.

Description of an object in position domain (physical, or material, world) is mostly related to the object's mobility in a reference frame (coordinate system) in the physical world. Thus, it comprises the three-dimensional vectors of position, velocity, acceleration, and a timestamp for which those vectors are valid, as outlined with (1).

$x_{\text {physical }}:=\{x, v, a, t\}$

The location domain stresses out the importance of relationship with the other related objects. Thus, the description of an object in the location domain provides the information needed for physical modelling of the object's shape, as well as those creating a context around the observed object, as presented with (2).

$x_{\text {contextual }}:=\{(p \quad$ ysical model $)($ contextual model $)\}(2)$

Relations between the objects are established through deployment of the concept of the physical distance. In practice, a three-dimensional Euclidean distance between two points $(\mathrm{x} 1, \mathrm{y} 1, \mathrm{z} 1)$ and $(\mathrm{x} 2, \mathrm{y} 2, \mathrm{z} 2)$ is commonly deployed, as defined with (3).

$d_{p \text { ysical }}=\sqrt{\left(x_{1}-x_{2}\right)^{2}+\left(y_{1}-y_{2}\right)^{2}+\left(z_{1}-z_{2}\right)^{2}}$

Contextual relationship with the other related objects is in focus, rather than physical properties of the object's dynamics. In due course, the information management differs according to the domain observed. While the information in the physical domain remains mostly of numerical nature, the location domain can and do introduce more complex non-numerical description of attributes, requiring complex database management, especially in the case of large data sets. Description of relationship between locations in location landscape (contextual world) are again based on distance, although the concept may be expanded vastly with combination of the physical distance and contextual (location distance) [3, 12], and utilisation of Tobler's First Law of Geography: 'Everything is related to everything else, but near things are more related than distant things. [13]. In the implementation of Tobler's First Law of Geography, proximity and distance may be measured in relation to contextual sense, such as using the information entropy measures [14]. Spatial statistical measures of relationship, spatial autocorrelation tests (Moran's I Test and Lagrange Multiplier Test) and spatial classification [3, 4], may be applied regardless of the initial distance definition.

Positioning and localisation, as the measurementfounded methods for estimation of the place of the observed object in the respective domains, develop the estimation uncertainties as unavoidable side effects. The positioning estimation uncertainty is commonly expressed using numerical error estimates, while localisation errors develop more complex nature driven by the properties of the contextual segment in question.

In general terms, the localisation errors are expressed more illustratively using parameters that describe the information lifetime, the level of trust in the information content, or the relevance to the contextual subset needed for a targeted LBS.

\section{Demonstration of the proposed concept}

Context-Matching method is a general term for a set of methods that match an experimental set of single-point positions establishing trajectory with the contextual details. Map-Matching method is an example of the class, a geometric method aims at improving position estimation through identification of usage of the known transport infrastructure (roads, railways, canals, utilisation of public transport etc.). It is frequently used to improve the satellite navigation position estimates through assessment of compliance with transport infrastructure for targeted groups of users.

Here we argue that Map-Matching (MM) method can be understood as a paradigm for a transformation between position and location domains. The MM method utilises the knowledge of position estimate of mobile objects and the knowledge of transport infrastructure. Both inputs are affected by measurement uncertainties and should be transferred into the same domain in order to allow for meaningful processing and provision of valuable services. The MM method transforms the inputs into the same domain, preserving the knowledge and creating the environment in which estimation error inherited from position domain (physical world) can be disregarded, providing they are not exceeding the level of QoS established for the observed family of LocationBased Services [1, 15]. For instance, the LBS that informs a mobile user about the approximate position of the nearest petrol station will work with the expected quality regardless of weather the position estimation is of the level of $0.2 \mathrm{~m}$, or $2 \mathrm{~m}$. 
Transformation using the MM method will reduce the overall space of contextual information to the level needed to satisfy the quality of the specific LocationBased Service [1]. The service QoS leveraging can be seen as the another advantage of utilization of the two domain approach in the LBS development [1]. We demonstrate the usefulness of the Map-Matching method in the case of matching the observed trajectory of walking along the promenade in the Old City of Baška, Krk Island, Croatia, with the known contextual data base (Open Street Map, 2021) with the results, as depicted in Figure 2.

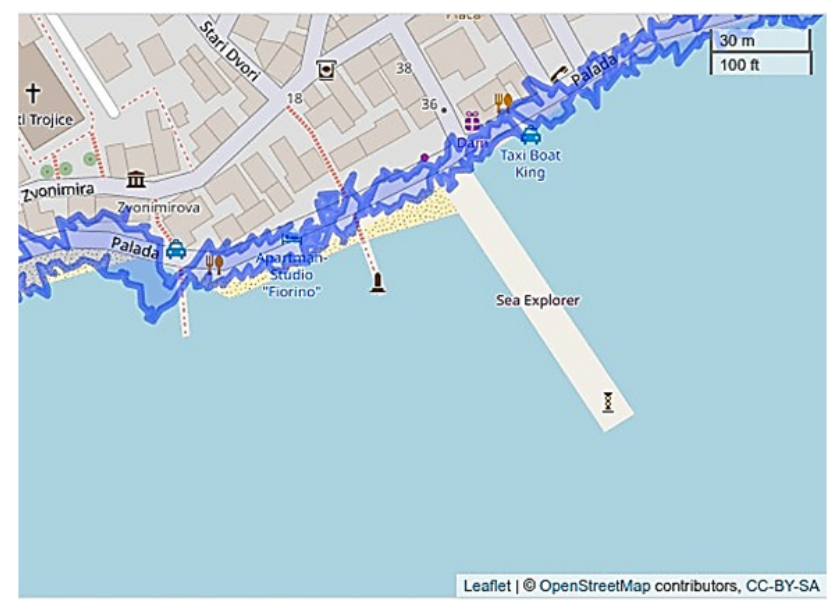

Figure 2. Matching GNSS-based trajectory observation with the actual contextual information.

Demonstration in Figure 2 is obtained using a tailored software developed in the $\mathrm{R}$ environment for statistical computing, its leaflet library, background map based on (Open Street Map, 2021) spatial data, and the experimental data collected by a Motorola moto One Action smartphone GNSS receiver in Summer 2019. Observed GNSS trajectory is depicted in terms of measurement uncertainty $( \pm 2 \sigma)$ around the estimated points.

\section{Conclusion}

The two domain (position- and location-based) model has proven capability of describing Location-Based Services formally. After defining the concept and the essential terms (position, location, positioning, and localization), here we provide formalism of the transition between the two domains, given in a sense of general transformation. The Map-Matching method considered an advanced positioning method, is proven to act as a transformation paradigm that connects the two domains, allowing for better understanding of the LBS development process, improved operations, and the more accurate determination of QoS or particular LocationBased Service.

Future work will concentrate on assembling an atlas of transformation functions to be utilized in the analysis and development of particular families (classes) of Location-Based Services.

\section{References}

[1] GSA. (2020). Report in Location-Based Services User Needs and Requirements. European GNSS Agency. Prague, Czechia. Available at:

https://www.gsceuropa.eu/sites/default/files/sites/all/files/Repor t_on_User_Needs_and_Requirements_LBS.pdf

[2] Filjar, R., Jezic, G., and Matijasevic, M. (2008). Location-Based Services: A Road Towards Situation Awareness. Journal of Navigation, 61, pp 573-589.

http://dx.doi.org/10.1017/S0373463308004864

[3] Loonis V., de Bellefon, M-P. (2018). Handbook of Spatial Analysis: Theory and Application with $R$. Insee Methodes, No.131. Insee \&Eurostat. Montrouge, France. Available at: https://www.insee.fr/en/information/3635545

[4] Efron, B., Hastie, T. (2016). Computer Age Statistical Inference: Algorithms, Evidence, and Data Science Institute of Mathematical Statistics Monographs. Cambridge: Cambridge University Press. doi:10.1017/CBO9781316576533

[5] Wiemann, S., Bernard, L. (2016). Spatial data fusion in Spatial Data Infrastructures using Linked Data. International Journal of Geographical Information Science, 30 (4), pp 613636. doi: 10.1080/13658816.2015.1084420

[6] Koks, D. (2007). Numerical Calculations for Passive Geolocation Scenarios. Australian Government, Department of Defence. Edinburgh, Australia.

[7] Frattasi, S., Della Rosa, F. (2017). Mobile Positioning and Tracking: From Conventional to Cooperative Techniques. John Wiley \& Sons. Chichester, UK.

[8] Filjar, R., Ševrović, M., Dadić, I. (2013). Positioning and localisation for Location-Based Services. Proc of 21st Telecommunications Forum TELFOR, Belgrade, Serbia, pp 1-4. doi: 10.1109/TELFOR.2013.6716158

[9] Filjar, R., Bušić, L., Dešić, S., Huljenić, D. (2008). LBS Position Estimation by Adaptive Selection of Positioning Sensors Based on Requested QoS. Chapter in: S Balandin et al. Next Generation Teletraffic and Wired/Wireless Advanced Networking. Lecture Notes in Computer Sciences, 5174, pp 101-109. Springer Verlag. Berlin and Heidelberg, Germany. doi: https://doi.org/10.1007/978-3-540-85500-2_9

[10] Filjar, R., Damas, M. C., Iliev, T. B. (2021). Resilient Satellite Navigation Empowers Modern Science, Economy, and Society. IOP Conf. Ser: Mater Sci Eng 1032, 012001, doi:10.1088/1757-899X/1032/1/012001

[11] Filjar, R., Sklebar, I., Horvat, M. (2020). A Comparison of Machine Learning-Based Individual Mobility Classification Models Developed on Sensor Readings from Loosely Attached Smartphones. Communications. Scientific Letters of the University of Žilina, 22(4), pp 153-162. doi: https://doi.org/10.26552/com.C.2020.4.153-162

[12] MacKay, D. J. C. (2003). Information Theory, Inference, and Learning Algorithms. Cambridge University Press. Cambridge, Available at:

http://www.inference.org.uk/itprnn/book.pdf

[13] Tobler, W. (1970). A computer movie simulating urban growth in the Detroit region. Economic Geography, 46(Supplement): pp 234-240.

[14] Gallotti, R., Bazzani, A., Degli Esposti, M., Rambaldi, S. (2013). Entropic measures of individual mobility patterns. Journal of Statistical Mechanics: Theory and Experiment, 2013, P10022 https://doi.org/10.1088/1742-5468/2013/10/P10022.

[15] ETSI. (2015). ETSI TS 103 246-1 (V1.1.1). Satellite Earth Stations and Systems (SES); GNSS based location systems; Part 1: Functional requirements. European Telecommunications Standardisation Institute. Sophia Antipolis, France.

[16] ETSI TR 121 900. (2021). Digital cellular telecommunications system, Phase 2+, GSM, (V16.4.0), https://www.etsi.org/deliver/etsi_tr/60/tr_121900v160400p.pdf 